\title{
Structure of a Liquid Crystalline Fluid around a Macroparticle: Density Functional Theory Study
}

\author{
David L. Cheung and Michael P. Allen \\ Department of Physics and Centre for Scientific Computing, \\ University of Warwick, Coventry, CV4 7AL, UK
}

\begin{abstract}
The structure of a molecular liquid, in both the nematic liquid crystalline and isotropic phases, around a cylindrical macroparticle, is studied using density functional theory. In the nematic phase the structure of the fluid is highly anisotropic with respect to the director, in agreement with results from simulation and phenomenological theories. On going into the isotropic phase the structure becomes rotationally invariant around the macroparticle with an oriented layer at the surface.
\end{abstract}

PACS numbers: 61.20.Gy,61.30.Cz,61.30.Jf

\section{INTRODUCTION}

Solid particles, both spherical and non-spherical, dispersed in a liquid crystal (LC) host comprise an interesting class of novel materials 1, 2]. In orientationally ordered phases of the host, the introduction of solid particles deforms the director field, leading to long-range interactions between the particles and effects such as chaining or the formation of soft solids. Colloidal dispersions in liquid crystals have a wide range of applications [3] and have recently attracted a great deal of interest [4].

Experimental techniques such as atomic force microscopy or confocal microscopy may be used to study LC-colloid dispersions; simulations and theory have also been applied. Simulations have been used to study the ordering of LC molecules around one [5, 6] or two macroparticles 7], and LC dispersions in confined geometries [8]. Phenomenological theories such as Landau-de Gennes [9, 10, 11, 12] or Frank elastic 13, 14] theory have also been used. These approaches however have their limitations: simulation is computationally expensive, while the aforementioned phenomenological theories require, often poorly known, parameters, and are incapable of accounting for spatial variation in the density (and, in the case of elastic theory, variation in the order parameter).

One popular theoretical method that may be applied to this type of problem is density functional theory (DFT) 15. Unlike Landau-de Gennes or elastic theory, this is capable of accounting for spatial variation in the density and order parameter and, in the form used here, requires knowledge only of the interaction potential between the molecules in the fluid. In this case DFT at the level of the Onsager second virial approximation 16] is applied to the case of a single, infinitely long, cylindrical macroparticle in a LC host. As the system is homogeneous along the length of the cylinder, two dimensions are sufficient to represent the spatial dependence of the density.

\section{THEORY}

For a system of uniaxial molecules the grand potential can be written as $\underline{17}$

$$
\beta \Omega[\rho(\boldsymbol{r}, \boldsymbol{u})]=\beta F_{\mathrm{id}}[\rho(\boldsymbol{r}, \boldsymbol{u})]+\beta F_{\mathrm{ex}}[\rho(\boldsymbol{r}, \boldsymbol{u})]+\beta \int \mathrm{d} \boldsymbol{r} \mathrm{d} \boldsymbol{u}\left(V_{\mathrm{ext}}(\boldsymbol{r}, \boldsymbol{u})-\mu\right) \rho(\boldsymbol{r}, \boldsymbol{u}),
$$

where $\rho(\boldsymbol{r}, \boldsymbol{u})$ is the position- and orientation-dependent single particle density, $V_{\text {ext }}(\boldsymbol{r}, \boldsymbol{u})$ is the external potential, $\mu$ is the chemical potential, and $\beta=1 / k_{B} T . F_{\text {id }}[\rho(\boldsymbol{r}, \boldsymbol{u})]$ and $F_{\mathrm{ex}}[\rho(\boldsymbol{r}, \boldsymbol{u})]$ are the ideal and excess free energies, respectively. $\boldsymbol{r}$ is the position vector and $\boldsymbol{u}$ is the orientation vector. The ideal free energy is given by

$$
\beta F_{\text {id }}[\rho(\boldsymbol{r}, \boldsymbol{u})]=\int \mathrm{d} \boldsymbol{r} \mathrm{d} \boldsymbol{u} \rho(\boldsymbol{r}, \boldsymbol{u})\{\log \rho(\boldsymbol{r}, \boldsymbol{u})-1\} .
$$

*Electronic address: david.cheung@warwick.ac.uk

${ }^{\dagger}$ Electronic address: m.p.allen@warwick.ac.uk 
The exact form of the excess free energy is generally unknown. Here we employ the Onsager approximation [16]

$$
\beta F_{\mathrm{ex}}[\rho(\boldsymbol{r}, \boldsymbol{u})]=-\frac{1}{2} \int \mathrm{d} \boldsymbol{r}_{1} \mathrm{~d} \boldsymbol{r}_{2} \mathrm{~d} \boldsymbol{u}_{1} \mathrm{~d} \boldsymbol{u}_{2} f\left(\boldsymbol{r}_{12}, \boldsymbol{u}_{1}, \boldsymbol{u}_{2}\right) \rho\left(\boldsymbol{r}_{1}, \boldsymbol{u}_{1}\right) \rho\left(\boldsymbol{r}_{2}, \boldsymbol{u}_{2}\right),
$$

where $f\left(\boldsymbol{r}_{12}, \boldsymbol{u}_{1}, \boldsymbol{u}_{2}\right)=\exp \left\{-\beta V\left(\boldsymbol{r}_{12}, \boldsymbol{u}_{1}, \boldsymbol{u}_{2}\right)\right\}-1$, the Mayer function, $\boldsymbol{r}_{12}=\boldsymbol{r}_{1}-\boldsymbol{r}_{2}$ and $V\left(\boldsymbol{r}_{12}, \boldsymbol{u}_{1}, \boldsymbol{u}_{2}\right)$ is the molecular pair interaction potential. The liquid crystal here is modelled as a fluid of prolate hard ellipsoids of elongation $e=a / b=5 ; a$ is the length of the symmetry, or major, axis and we will use the minor axis $b=1$ as a unit of length. When two molecules overlap, $V=\infty$ and $f=-1$; for a non-overlapping pair, $V=0$ and $f=0$. The approximation of eqn (3) corresponds to truncating the virial expansion after the pair term. While Onsager theory is exact only in the limit of infinite elongation, it has been used to study the anchoring of ellipsoids of this elongation near solid substrates [18, 19] and has been found to give results in qualitative agreement with simulation.

The external potential, representing a single cylindrical macroparticle of radius $R$ oriented along the $y$ axis, is given by

$$
V_{\text {ext }}(\boldsymbol{r}, \boldsymbol{u})=V_{\text {ext }}(\boldsymbol{s}, \boldsymbol{u})= \begin{cases}\frac{1}{2} V_{0}[\tanh (b / w)-\tanh (-b / w)] & s-R<-b \\ \frac{1}{2} V_{0}\left[\tanh \left(\frac{R-s}{w}\right)-\tanh (-b / w)\right] & |s-R|<b \\ 0 & s-R>b\end{cases}
$$

where $s=(x, z), s=|s|, V_{0}=50 k_{B} T$ and $w=b / 5$. This represents a sharply varying repulsive potential acting on the ellipsoid centres of mass. It excludes the molecules from the cylinder and gives rise to homeotropic (normal) anchoring at the surface.

As before [20, 21, 22, 23] the angularly dependent functions are expanded in a set of spherical harmonics, and the assumption of translational invariance along $y$ allows us to write the coefficients as functions of $s$ :

$$
\begin{aligned}
\log \rho(\boldsymbol{r}, \boldsymbol{u}) & =\sum_{\ell, m} \tilde{\rho}_{\ell m}(\boldsymbol{s}) Y_{\ell m}(\boldsymbol{u}) \\
\rho(\boldsymbol{r}, \boldsymbol{u}) & =\sum_{\ell, m} \rho_{\ell m}(\boldsymbol{s}) Y_{\ell m}^{*}(\boldsymbol{u}) \\
V_{\mathrm{ext}}(\boldsymbol{r}, \boldsymbol{u}) & =\sum_{\ell, m} V_{\ell m}(\boldsymbol{s}) Y_{\ell m}(\boldsymbol{u}) .
\end{aligned}
$$

Note the complex conjugate in the density expansion. The Mayer function is expanded as [22]

$$
f\left(\boldsymbol{r}_{12}, \boldsymbol{u}_{1}, \boldsymbol{u}_{2}\right)=\sum_{\ell_{1}, \ell_{2}, \ell} f_{\ell_{1} \ell_{2} \ell}\left(r_{12}\right) \Phi_{\ell_{1} \ell_{2} \ell}\left(\hat{\boldsymbol{r}}_{12}, \boldsymbol{u}_{1}, \boldsymbol{u}_{2}\right)
$$

where $r_{12}=\left|\boldsymbol{r}_{12}\right|, \hat{\boldsymbol{r}}_{12}=\boldsymbol{r}_{12} / r_{12}$, and $\Phi_{\ell_{1} \ell_{2} \ell}$ is a rotational invariant [24]. Inserting these expressions into the grand potential and integrating over angles and the $y$ direction gives

$$
\begin{aligned}
\frac{\beta \Omega[\rho(\boldsymbol{r}, \boldsymbol{u})]}{L} & =\int \mathrm{d} \boldsymbol{s} \sum_{\ell, m} \rho_{\ell m}(\boldsymbol{s})\left(\tilde{\rho}_{\ell m}(\boldsymbol{s})-\sqrt{4 \pi}(1+\beta \mu) \delta_{l 0}+\beta V_{\ell m}(\boldsymbol{s})\right) \\
& +\int \mathrm{d} \boldsymbol{s}_{1} \mathrm{~d} \boldsymbol{s}_{2} \sum_{\substack{\ell_{1} m_{1} \\
\ell_{2} m_{2}}} \mathcal{L}_{\ell_{1} m_{1} \ell_{2} m_{2}}\left(\boldsymbol{s}_{12}\right) \rho_{\ell_{1} m_{1}}\left(\boldsymbol{s}_{1}\right) \rho_{\ell_{2} m_{2}}\left(\boldsymbol{s}_{2}\right) .
\end{aligned}
$$

Here $L$ is the box length in the $y$ direction (we assume periodicity). The quantities $\mathcal{L}_{\ell_{1} m_{1} \ell_{2} m_{2}}\left(\boldsymbol{s}_{12}\right)$ come from integrating the Mayer function and are the spherical harmonic coefficients of the excluded length (in the $y$ direction) of two molecules with a separation vector $s_{12}=s_{1}-s_{2}$ in the $x z$-plane, treated as a function of the molecular orientations. As the last term in eqn (7) is a convolution, it is most conveniently evaluated in reciprocal space. If $\rho_{l m}(\boldsymbol{k})$ is the two-dimensional Fourier transform of $\rho_{l m}(\boldsymbol{s})$ then this term may be written

$$
\sum_{\boldsymbol{k}} \sum_{\substack{\ell_{1} m_{1} \\ \ell_{2} m_{2}}} \mathcal{L}_{\ell_{1} m_{1} \ell_{2} m_{2}}(\boldsymbol{k}) \rho_{\ell_{1} m_{1}}(\boldsymbol{k}) \rho_{\ell_{2} m_{2}}(\boldsymbol{k})
$$

where $\mathcal{L}_{\ell_{1} m_{1} \ell_{2} m_{2}}(\boldsymbol{k})$ is the Fourier transform of $\mathcal{L}_{\ell_{1} m_{1} \ell_{2} m_{2}}\left(\boldsymbol{s}_{12}\right)$. 
In order to find the equilibrium density the functions are tabulated on a regular grid in the $x z$ plane; the grid spacing is $\delta x=\delta z=0.2 b$, the molecular length corresponding to 25 grid points. The grand potential is then minimised with respect to the $\tilde{\rho}_{l m}(s)$ coefficients at each grid point using the conjugate gradient method 25]. When required, the coefficients $\rho_{\ell m}(s)$ are calculated through eqns (5), with angular integrations performed using Lebedev quadrature [26, 27]. The systems in studied in this work were all square boxes $\left(L_{x}=L_{z}\right)$ with $L_{x}$ ranging from $40 b$ (200 grid points) to $100 b$ (500 grid points). Table $\square$ summarises the parameters of the systems discussed in detail in the next section.

\begin{tabular}{cccc}
\hline$R / b$ & $L_{x} / b$ & $n_{x}$ & $\delta k_{x} / b^{-1}$ \\
\hline 5 & 40 & 200 & $\pi / 20$ \\
7.5 & 60 & 300 & $\pi / 30$ \\
10 & 60 & 300 & $\pi / 30$ \\
15 & 80 & 400 & $\pi / 40$ \\
20 & 100 & 500 & $\pi / 50$ \\
\hline
\end{tabular}

TABLE I: Details of systems studied. $R$ is the macroparticle radius, $L_{x}$ is the box length in the $x$ direction (with $L_{z}=L_{x}$ ), $n_{x}$ is the number of grid points in the $x$ direction and $\delta k_{x}$ is the grid spacing in $k$ space.

Once the equilibrium density coefficients $\rho_{\ell m}(s)$ have been determined, the number density $\rho(s)$ around the macroparticle may be found from

$$
\rho(\boldsymbol{s})=\int \mathrm{d} \boldsymbol{u} \rho(\boldsymbol{s}, \boldsymbol{u})=\sqrt{4 \pi} \rho_{00}(\boldsymbol{s}) .
$$

The orientational ordering is described by the order tensor $Q_{\alpha \beta}(\boldsymbol{s})$ that is found from $\rho(\boldsymbol{s}, \boldsymbol{u})$ by

$$
Q_{\alpha \beta}(\boldsymbol{s})=\frac{3}{2} \int \mathrm{d} \boldsymbol{u} \rho(\boldsymbol{s}, \boldsymbol{u}) u_{\alpha}(\boldsymbol{s}) u_{\beta}(\boldsymbol{s})-\frac{1}{2} \delta_{\alpha \beta}, \quad \alpha, \beta=x, y, z .
$$

The spatially varying order parameter $S(\boldsymbol{s})$ is given by the largest eigenvalue of $Q_{\alpha \beta}(\boldsymbol{s})$ and the director $\boldsymbol{n}(\boldsymbol{s})$ by the eigenvector associated with $S(s)$.

\section{RESULTS}

\section{A. Structure in the nematic phase}

First we examine the fluid structure around the cylindrical particle in a nematic fluid, at chemical potential $\mu=8.0$. The density distributions around cylinders of radius $5 \leq R / b \leq 20$ are shown in Fig. 1 For all $R$ the density is largest at the surface but then decays away with almost periodic variation, similar to that seen for a nematic-planar wall interface. Further from the cylinder this distortion in the density becomes highly anisotropic. Parallel to the director the modulations in the density are stronger than in the perpendicular direction. This weakening perpendicular to the director is due to the partial melting of the nematic in the defect regions [28] and is most noticeable for the smallest radius $R / b=5$ (Fig. 1 $\mathrm{T}$ ). In this case, there is almost no density modulation perpendicular to the director causing the density map to show chevron-like structures, with the chevron tips pointing along the director away from the axis of the cylinder. For larger radii, the density variation perpendicular to the director is stronger, but shorter-ranged, than in the direction parallel to the director. In comparison to simulation of a system with $e=3$ [28], the density variation seems to be shorter ranged. While this may be due to differences in the models, studies of the nematic-wall interface using Onsager and related theories [18, 23. also gave density profiles that have generally weaker structure than comparable simulations.

Maps of the orientational order parameter around the cylindrical particles are shown in Fig. 2 As for the density maps, the variation in the order parameter is highly anisotropic. For $R / b=5$, along the director, there are lobes of high order along the top and bottom of the cylinder. In the direction perpendicular to the director there are two regions of drastically reduced order, corresponding to defects in the liquid. As $R$ increases the lobes of increased order tend to wrap around the cylinder, and the defects move away from the surface, in agreement with simulation [28] and phenomenological theory [11, 29, 30]. While the positions of the defects are in qualitative agreement with previous results, in the present case the defects are significantly smaller than those seen previously, suggesting that for molecules of this elongation more sophisticated methods such as weighted density approximations [31] or fundamental 

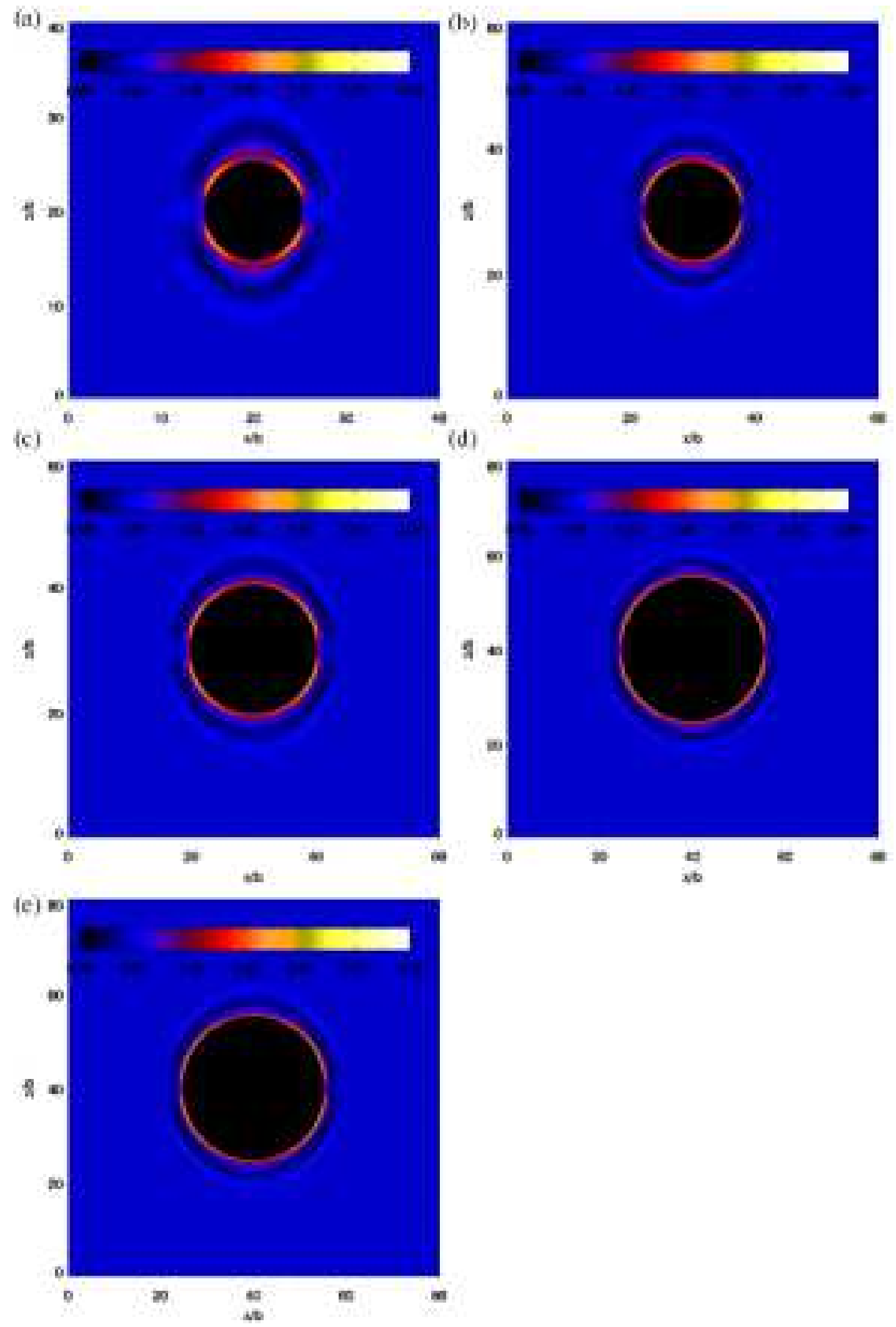

FIG. 1: Density maps around a cylindrical macroparticle at $\mu=8.0$ (nematic phase). Cylinder radius $R / b$ : (a) 5.0, (b) 7.5, (c) 10.0, (d) 15.0, and (e) 20.0. Dark colours show areas of low $\rho(s)$, light colours high $\rho(s)$. 

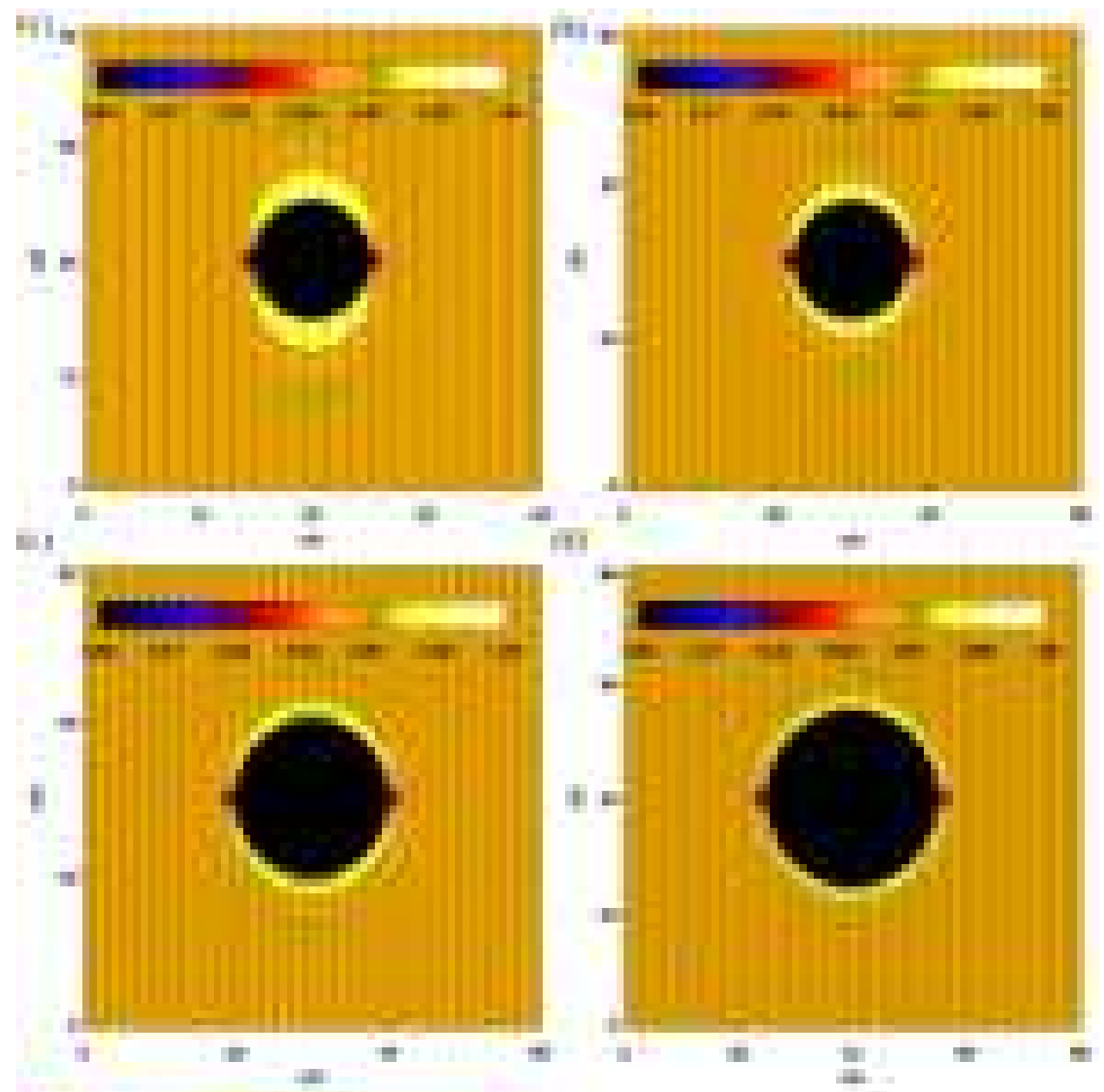

III
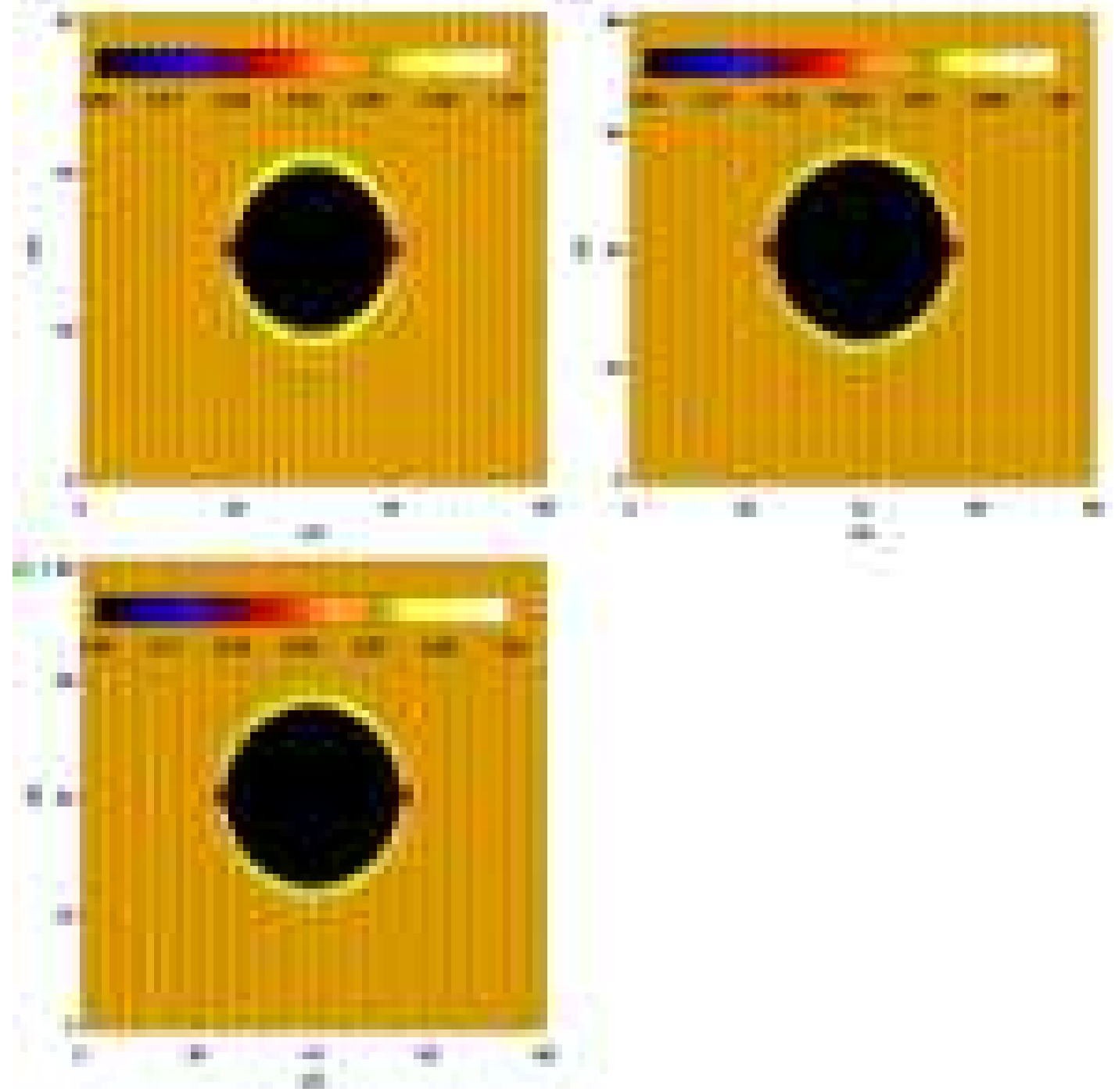

FIG. 2: Order parameter maps around a cylindrical macroparticle at $\mu=8.0$ (nematic phase). Cylinder radius $R / b$ : (a) 5.0, (b) 7.5, (c) 10.0, (d) 15.0, and (e) 20.0. Dark colours show areas of low $S(s)$, light colours high $S(s)$. Solid lines show the orientation of the local director $n(s)$. 
measure theory [32] are necessary to examine the structure within the topological defect. Detailed comparisons with simulation will appear in a later publication.

Also shown in Fig. 2 is the director orientation $\boldsymbol{n}(\boldsymbol{s})$ around the macroparticle. At large distances from the cylinder the $\boldsymbol{n}(\boldsymbol{s})$ lies along the $z$ axis. Close to the particle the director becomes highly distorted. Along the $z$ axis and at the defects $\boldsymbol{n}(\boldsymbol{s})$ is normal to the particle surface. At other points on the particle surface, $\boldsymbol{n}(\boldsymbol{s})$ points away from the surface normal appearing to graze the surface of the macroparticle. This behaviour is different from that seen in simulation [28] and from elastic theory in the case of strong anchoring [29], suggesting that the anchoring around the macroparticle is weaker than in previous studies. Apart from in the vicinity of the $\operatorname{defects} \boldsymbol{n}(\boldsymbol{s})$ is a smoothly varying function of $s$.

In order to gain more insight, the density and order parameter profiles parallel $\left(\rho_{\|}(s)\right.$ and $\left.S_{\|}(s)\right)$ and perpendicular $\left(\rho_{\perp}(s)\right.$ and $\left.S_{\perp}(s)\right)$ to the director (through the defects) are shown in Fig. 3 The $\rho_{\|}(s)$ curves show similar structure
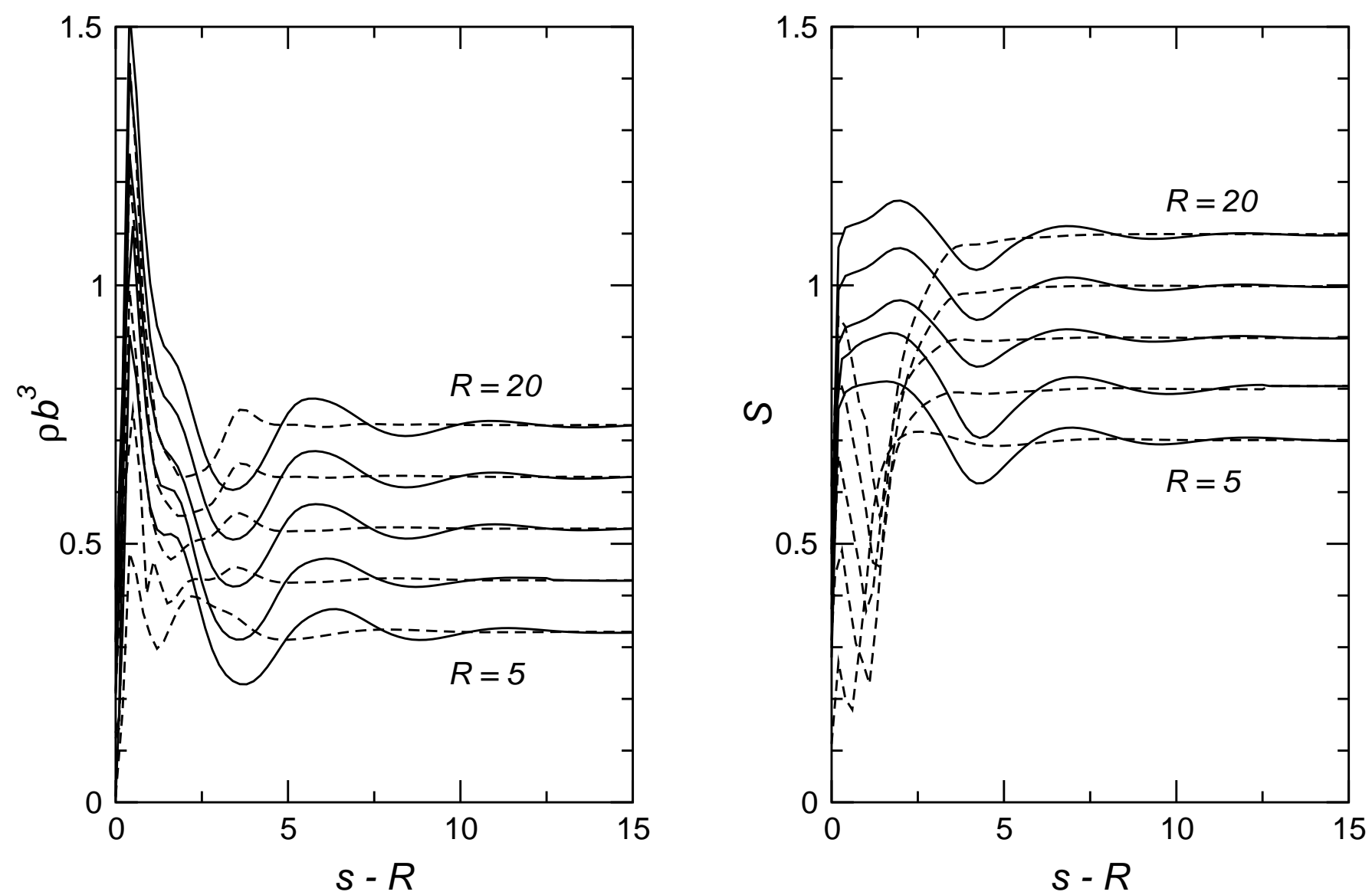

FIG. 3:

(a) Density profiles, $\rho(s) b^{3}$, and (b) order parameter profiles $S(s)$, around a cylindrical macroparticle at $\mu=8.0$ (nematic phase). Full lines: in the direction parallel to the director. Dashed lines: in the direction perpendicular to the director. Results are shown for cylinder radius $R / b=5.0$ and, displaced successively upwards by 0.1 units for clarity, $R / b=7.5,10.0,15.0,20.0$.

as $R$ increases, with a peak at the cylinder surface $s=R$, a second peak about $5 b$ (one molecular length) from the surface, and some decaying oscillations into the bulk. This is similar to the behaviour seen for a nematic fluid near a wall with homeotropic alignment $[20,23]$. The size of the peak at contact increases with $R$ at small $R . \rho_{\perp}(s)$ also has a peak at contact. For smaller cylinders $\rho_{\perp}(R) \ll \rho_{\|}(R)$. As $R$ increases the difference between the two heights decreases. Further from contact $\rho_{\perp}(s)$ has a secondary peak. For the $R / b=5$ cylinder this peak is approximately $2.5 b$ from the surface with $\rho_{\perp}(s)$ tending towards the bulk value further out. For larger $R$, the secondary peak moves out. Beyond this peak $\rho_{\perp}(s)$ behaves almost identically for all $R$.

As with $\rho_{\|}(s)$, the $S_{\|}(s)$ profile is similar to the order parameter profile for a nematic-homeotropic wall interface, with a maximum at contact and decaying oscillations. $S_{\perp}(r)$ shows a minimum near contact. This corresponds to the defect seen in the order parameter maps (Fig. 21). In agreement with the order parameter maps, the distance between the minimum and the surface increases with $R$. Shown in Fig. 团is the position of the minimum $s_{\min }$ as a function of 
radius $R$. For spherical macroparticles with both Saturn ring and satellite defects, the distance between the surface

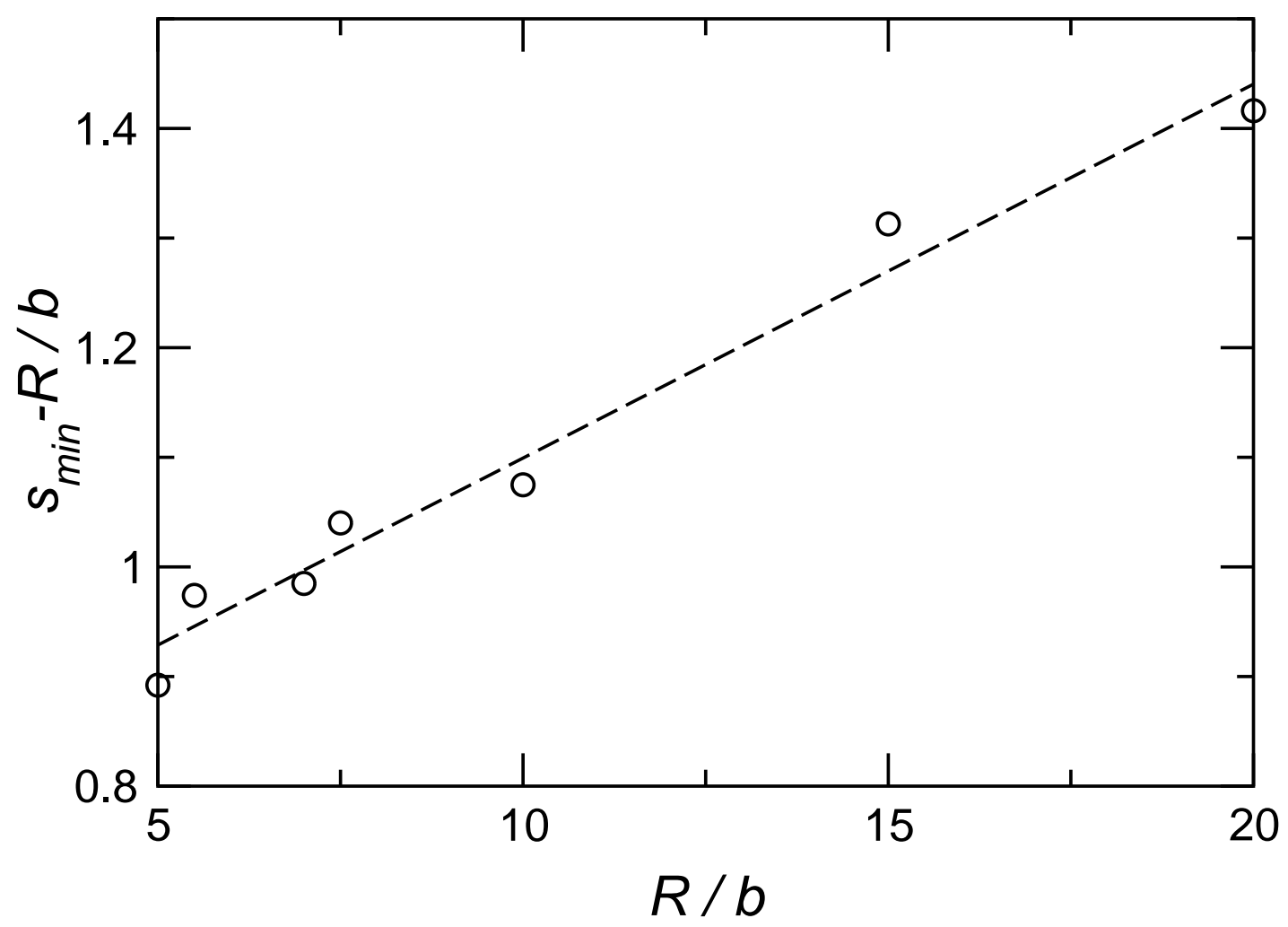

FIG. 4: Defect position $\left(s_{\text {min }}-R\right)$ near cylindrical macroparticle at $\mu=8.0$ (nematic phase) as a function of radius $R$. Dashed line shows the line of best fit.

and the defect varies linearly with particle size [6]. In the present case $s_{\text {min }}$ increases with $R$.

\section{B. Structure in the isotropic phase and around the nematic-isotropic transition}

Shown in Figs. 11, 2], 5 and 6 are the density and order parameter maps for a cylindrical macroparticle with $R / b=15$ in both the nematic and isotropic phases. The bulk isotropic-nematic transition occurs at $\mu_{N I}=7.3675$. In the nematic phase, at this value of $\mu$, the density and order parameter maps are similar to those seen for $\mu=8$, though the variation in the density and order parameter are less pronounced. Also the defects either side of the cylinder with $\mu=7.3675$ are both larger and significantly further from the surface $\left(s_{\min }-R \approx 2.5 b\right)$ than for the higher chemical potential. In the isotropic phase, both the density and order parameter variation become almost completely symmetrical, reflecting the loss of a preferred direction. In this phase, far from the cylinder, the order parameter $S \rightarrow 0$, while both $\rho$ and $S$ have maxima at the surface. This suggests that the surface is wet by the nematic and this gives rise to short range interactions between particles dispersed in a liquid crystal host even in the isotropic phase [9, 33]. However, a detailed investigation of surface phase behaviour and interparticle interactions in this model await further study.

\section{CONCLUSIONS}

In this paper the structure of a liquid of hard ellipsoids of elongation $e=5$, around a cylindrical macroparticle, in both the nematic and isotropic phases, was studied using density functional theory within the Onsager approximation. The resulting density and order parameter maps were consistent with previous theoretical and simulation work. On going from the nematic to isotropic phase, the structure of the surrounding fluid becomes rotationally invariant about the cylinder, with what appears to be a nematic wetting layer at the particle surface.

The present study is preliminary: investigation of the sensitivity of the results to the resolution of the real-space and reciprocal-space grids must still be carried out. Nonetheless, the results are very promising. Despite its simplicity, 

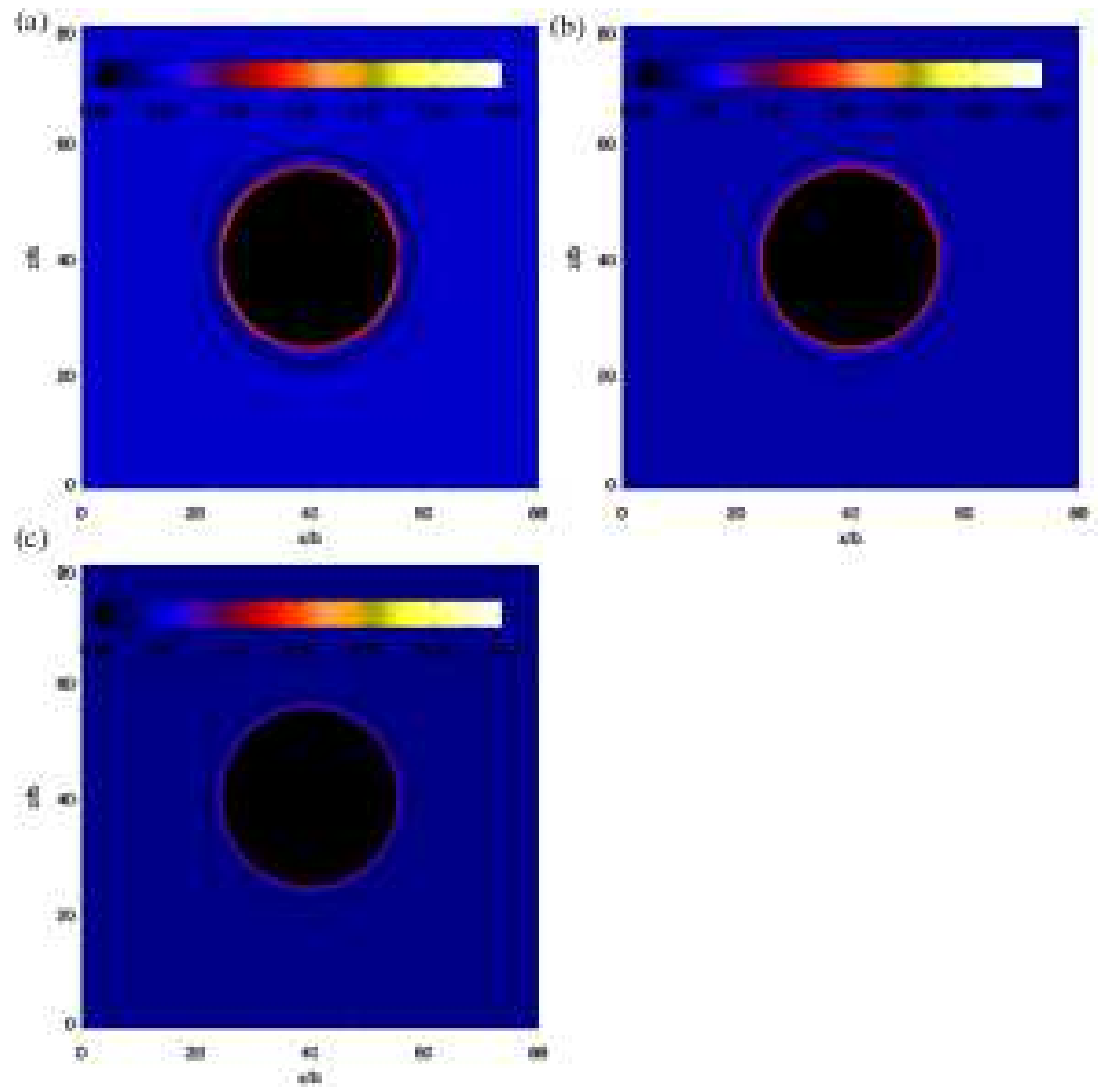

FIG. 5: Density maps for fluid around cylindrical macroparticle of radius $R / b=15$ with (a) $\mu=7.3675$ (nematic phase, at NI transition), (b) $\mu=7.3675$ (isotropic phase, at NI transition), and (c) $\mu=5.0$ (isotropic phase). Dark colours show low $\rho(s)$, light colours high $\rho(s)$.

this method provides results that are generally in agreement with simulation and phenomenological theory. The only major deficiency is that the defects are smaller than would be expected from simulations of a similar system with $e=3$. Using more sophisticated density functionals or ellipsoids with longer elongations (where Onsager theory is more accurate) would hopefully give a better description of the defect. Detailed comparisons with simulation will be described elsewhere.

\section{Acknowledgments}

This research was supported by EPSRC grant GR/S77240. The calculations were performed on the computing facilities of the Centre for Scientific Computing, University of Warwick. The authors of Ref. 15] are thanked for providing a preprint.

[1] P. Poulin, Curr. Opin. Colloid Interf. Sci. 4, 66 (1999). 

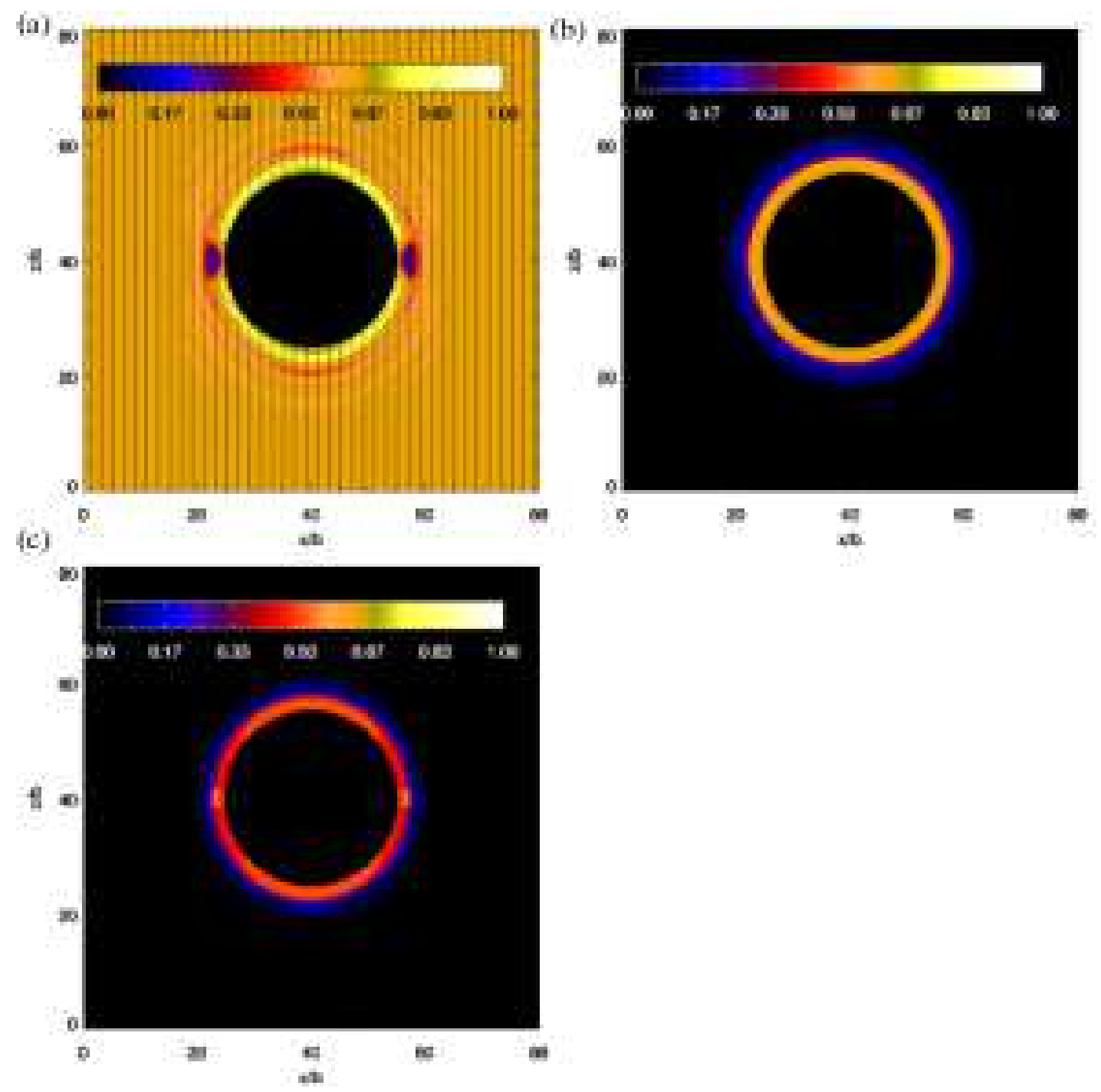

FIG. 6: Order parameter maps for fluid around cylindrical macroparticle of radius $R / b=15$ with (a) $\mu=7.3675$ (nematic phase, at NI transition), (b) $\mu=7.3675$ (isotropic phase, at NI transition), and (c) $\mu=5.0$ (isotropic phase). Dark colours show areas of low $S(s)$, light colours high $S(s)$.

[2] H. Stark, Physics Reports 351, 387 (2001).

[3] W. B. Russel, D. A. Saville, and W. R. Schowalter, Colloidal dispersions (Cambridge University Press, Cambridge, 1989).

[4] J. Phys. Cond. Mat. 16 (2004), special issue containing the proceedings of an ESF PESC exploratory workshop on Liquid Crystal Colloid Dispersions, 2004.

[5] J. L. Billeter and R. A. Pelcovits, Phys. Rev. E 62, 711 (2000).

[6] D. Andrienko, G. Germano, and M. P. Allen, Phys. Rev. E 63, 041701/1 (2001).

[7] M. S. Al-Barwani, G. S. Sutcliffe, and M. P. Allen, J. Phys. Chem. B 108, 6663 (2004).

[8] E. B. Kim, R. Faller, Q. Yan, N. L. Abbot, and J. J. de Pablo, J. Chem. Phys. 117, 7781 (2002).

[9] A. Borštnik, H. Stark, and S. Žumer, Phys. Rev. E 60, 4210 (1999).

[10] P. Patrício, M. Tasinkevych, and M. M. Telo da Gama, Euro. Phys. J. E 7, 117 (2002).

[11] M. Tasinkevych, N. M. Silvestre, P. Patrício, and M. M. Telo da Gama, Euro. Phys. J. E 9, 341 (2002).

[12] D. Andrienko, M. Tasinkevych, P. Patrício, and M. M. T. da Gama, Phys. Rev. E 69, 021706/1 (2004).

[13] H. Stark, Euro. Phys. J. B 10, 311 (1999).

[14] R. Yamamoto, Y. Nakayama, and K. Kim, J. Phys. Cond. Mat. 16, S1945 (2004).

[15] L. Harnau and S. Dietrich, in Soft Matter, edited by G. Gompper and M. Schick (Wiley-VCH, Berlin, 2006), vol. 3, to appear.

[16] L. Onsager, Ann. N. Y. Acad. Sci. 51, 627 (1949).

[17] J.-P. Hansen and I. R. McDonald, Theory of Simple Liquids (Academic Press, London, 1986), 2nd ed. 
[18] A. Chrzanowska, P. I. C. Teixeira, H. Ehrentraut, and D. J. Cleaver, J. Phys. Cond. Mat. 13, 4715 (2001).

[19] P. I. C. Teixeira, F. Barmes, and D. J. Cleaver, J. Phys. Cond. Mat. 16, S1969 (2004).

[20] M. P. Allen, Molec. Phys. 96, 1391 (1999).

[21] M. P. Allen, J. Chem. Phys. 112, 5447 (2000).

[22] D. Andrienko and M. P. Allen, Phys. Rev. E 65, 021704/1 (2002).

[23] D. L. Cheung and F. Schmid, J. Chem. Phys. 120, 9185 (2004).

[24] C. G. Gray and K. E. Gubbins, Theory of molecular fluids. 1. Fundamentals (Clarendon Press, Oxford, 1984).

[25] W. H. Press, B. P. Flannery, S. A. Teukolsky, and W. T. Vetterling, Numerical Recipes (Cambridge University Press, Cambridge, 1986)

[26] V. I. Lebedev, Zh. Vychisl. Mat. Mat. Fiz 16, 293 (1976), (English translation: USSR Comput. Math. \& Math. Phys. 16, $10(1976))$.

[27] V. I. Lebedev, Sibirskii Matematicheskii Zhurnal 18, 132 (1977).

[28] D. Andrienko, M. P. Allen, G. Skačej, and S. Žumer, Phys. Rev. E 65, 041702/1 (2002).

[29] S. V. Burylov and Y. L. Raikher, Phys. Rev. E 50, 358 (1994).

[30] J. Fukuda and H. Yokoyama, Euro. Phys. J. E 4, 389 (2001).

[31] A. M. Somoza and P. Tarazona, J. Chem. Phys. 91, 517 (1989).

[32] G. Cinacchi and F. Schmid, J. Phys. Cond. Mat. 14, 12223 (2002).

[33] K. Kočevar and I. Muševič, Phys. Rev. E 64, 051711/1 (2001). 